\title{
OSCILLATION OF A FUNCTIONAL DIFFERENTIAL EQUATION ARISING FROM AN INDUSTRIAL PROBLEM
}

\author{
HIROSHI ONOSE \\ (Received 1 April 1976; revised 7 June 1977) \\ Communicated by $\mathrm{N}$. Trudinger
}

\begin{abstract}
In the last few years, the oscillatory behavior of functional differential equations has been investigated by many authors. But much less is known about the first-order functional differential equations. Recently, Tomaras (1975b) considered the functional differential equation

$$
y^{\prime}(t)=\sum_{i=1}^{n} p_{i}(t) y\left(g_{i}(t)\right)+q(t) y(t)+r(t),
$$

and gave very interesting results on this problem, namely the sufficient conditions for its solutions to oscillate. The purpose of this paper is to extend and improve them, by examining the more general functional differential equation
\end{abstract}

$$
y^{\prime}(t)=\sum_{i=1}^{n} p_{i}(t) f\left(y\left(\dot{g}_{i}(t)\right)\right)+q(t) y(t)+r(t)
$$

Subject classification (Amer. Math. Soc. (MOS) 1970): 34 C 10.

\section{Introduction}

The first-order functional differential equation

$$
y^{\prime}(t)=a y(\lambda t)+b y(t)
$$

arises from an industrial problem. This equation has been discussed in detail by Kato (1972) and Kato and McLeod (1971), and its oscillatory behavior (for $b=0$ ) has been mentioned by Ladas, Lakshmikantham and Papadakis (1972). The oscillatory behavior of the more general functional differential equation

$$
y^{\prime}(t)=\sum_{i=1}^{n} p_{i}(t) y\left(g_{i}(t)\right)+q(t) y(t)
$$


was examined by Tomaras ((1975a) for $n=1),(1975 b$, and results obtained were extended, by Tomaras ((1975a) for $n=1),(1975 b)$ also, to the more general functional differential equation with forcing term

$$
y^{\prime}(t)=\sum_{i=1}^{n} p_{i}(t) y\left(g_{i}(t)\right)+q(t) y(t)+r(t) .
$$

Here, we will extend Tomaras's results for (2) and (3), by studying the oscillatory behavior of the more general equations

$$
y^{\prime}(t)=\sum_{i=1}^{n} p_{i}(t) f\left(y\left(g_{i}(t)\right)\right)+q(t) y(t)
$$

and

$$
y^{\prime}(t)=\sum_{i=1}^{n} p_{i}(t) f\left(y\left(g_{i}(t)\right)\right)+q(t) y(t)+r(t) .
$$

In what follows, a solution of (4) or (5) is said to be "oscillatory", if it has arbitrarily large zeros, and "nonoscillatory" otherwise.

The author wishes to express his thanks to the referee for some very useful comments.

\section{Unforced oscillation}

We state at first the following lemma, which is an extension of Tomaras's (1975b) Lemma 2.1.

LEMMA 1. Consider the functional differential equation

$$
y^{\prime}(t)+\sum_{i=1}^{n} F_{i}(t) h\left(y\left(g_{i}(t)\right)\right)=0,
$$

subject to the following conditions:

(C1) $F_{i}(t), g_{i}(t) \in C[[0, \infty), R], F_{i}(t) \geqslant 0, i=1,2, \ldots, n$;

(C2) $g_{i}(t)<t, \lim _{t \rightarrow \infty} g_{i}(t)=\infty$ and $g_{i}(t)$ are nondecreasing, $i=1,2, \ldots, n$;

(C3) $h(y) \in C[R, R], y h(y)>0(y \neq 0), h(y)$ is nondecreasing and $h(x y)=h(x) h(y)$ for $x, y \in R$.

If, in addition,

$$
\lim _{t \rightarrow \infty} \sup \sum_{i=1}^{n} \int_{G(i)}^{t} F_{i}(s) d s>M
$$


and

$$
\lim _{y \rightarrow 0} \frac{|y|}{h(|y|)} \leqslant M
$$

where $M$ is a positive constant and $G(t)=\max g_{i}(t)(i=1,2, \ldots, n)$, then every solution of $(6)$ is oscillatory.

Proof. Suppose that there exists a nonoscillatory solution $y(t)$ of (6). Without loss of generality, we may suppose that $y(t)>0$ for $t \geqslant t_{0}$, where $t_{0}$ is a sufficiently large constant. From (6) because of $(\mathrm{C} 1),(\mathrm{C} 2),(\mathrm{C} 3)$, we have $y^{\prime}(t) \leqslant 0$. Integrating (6) from $G(t)$ to $t$, we have

$$
y(t)-y(G(t))+\int_{G(t)}^{t} \sum_{i=1}^{n} F_{i}(s) h\left(y\left(g_{i}(s)\right)\right) d s=0 .
$$

From this and $y^{\prime}(t) \leqslant 0$, we have

$$
y(t)-y(G(t))+\sum_{i=1}^{n} h(y(G(t))) \int_{a(t)}^{t} F_{i}(s) d s \leqslant 0 .
$$

By $y^{\prime}(t) \leqslant 0$, we have

$$
\lim _{t \rightarrow \infty} y(t)=c,
$$

where $c \geqslant 0$ is a constant. Suppose $c>0$; then, because of (7), (9) leads to a contradiction. Now, suppose $c=0$. From (9), we have

$$
0 \leqslant \sum_{i=1}^{n} \int_{Q(t)}^{t} F_{i}(s) d s \leqslant \frac{y(G(t))}{h(y(G(t)))} .
$$

If we take the lim sup of (11), as $t \rightarrow \infty$, because of (8), we get a contradiction to (7).

In the case where the assumption (8) does not hold, it is easy to verify the following

LEMMA 2. Suppose that the conditions (C1), (C2), (C3) and (7) hold. Then, every solution $y(t)$ of $(6)$ is oscillatory or $\lim _{l \rightarrow \infty} y(t)=0$.

Now make the transformation $z(t)=y(t) \exp \left(-\int_{c^{*}}^{t} q(s) d s\right)$, where $c^{*}$ is a constant, to get (4) in the form

$$
z^{\prime}(t)+\sum_{i=1}^{n} l_{i}(t) f\left(z\left(g_{i}(t)\right)\right)=0
$$

where

$$
l_{i}(t)=-p_{i}(t) f\left(\exp \left(\int_{c^{*}}^{g_{i}(t)} q(s) d s\right)\right) \exp \left(-\int_{c^{*}}^{t} q(s) d s\right), \quad i=1,2, \ldots, n .
$$


This transformation transforms (4) to (12), provided that (C3) $(f(x y)=f(x) f(y))$ holds. This is of the same form as (6) and so, applying Lemma 1 to it, we have the following

THEOREM 1. Consider the functional differential equation (4), subject to the following conditions:

(i) $p_{i}(t), g_{i}(t) \in C[[0, \infty), R],-p_{i}(t) \geqslant 0, i=1,2, \ldots, n$;

(ii) $g_{i}(t)<t, \lim _{i \rightarrow \infty} g_{i}(t)=\infty$ and $g_{i}(t)$ are nondecreasing, $i=1,2, \ldots, n$;

(iii) $q(t)$ is continuous for any $t \neq 0$;

(iv) $f(y) \in C[R, R], y f(y)>0(y \neq 0), f(y)$ is nondecreasing and $f(x y)=f(x) f(y)$ for $x, y \in R$.

If, in addition,

(13) $\limsup _{t \rightarrow \infty} \sum_{i=1}^{n} \int_{G(i)}^{l}-p_{i}(s) f\left(\exp \left(\int_{c^{*}}^{g_{i}(s)} q(u) d u\right)\right) \exp \left(-\int_{c^{*}}^{s} q(u) d u\right) d s>M$,

where $G(t)=\max g_{i}(t), i=1,2, \ldots, n$, and $M$ is a positive constant, and

$$
\lim _{y \rightarrow 0} \frac{|y|}{f(|y|)} \leqslant M,
$$

then, every solution of (4) is oscillatory.

Remark. Theorem 1 contains Tomaras's (1975b) Theorem 2.1.

In the case where the assumption (14) does not hold, we get the following

THEOREM 2. Suppose that conditions (i), (ii), (iii), (iv) and (13) are satisfied for (4). Then, every solution $y(t)$ of (4) is oscillatory or

$$
\lim _{t \rightarrow \infty} y(t) \exp \left(-\int_{c^{*}}^{t} q(s) d s\right)=0 .
$$

The proof follows from Lemma 2.

EXAMPLE. Consider the equation

$$
y^{\prime}(t)+\frac{1}{2}\left(\frac{t-15}{t-5}\right)^{3 / 2} y^{3}(t-10)=0 .
$$

It is easily verified that the conditions of Lemma 2 are satisfied. Especially, for (7), we have

$$
\limsup _{t \rightarrow \infty} \int_{t-10}^{t} \frac{1}{2}\left(\frac{s-15}{s-5}\right)^{3 / 2} d s>\lim _{t \rightarrow \infty} \sup \int_{t-10}^{t} \frac{1}{2}\left(\frac{1-15 / s}{1-5 / s}\right)^{3 / 2} d s>\frac{10}{4} .
$$

Clearly, the condition (8) of Lemma 1 does not hold and (15) has a nonoscillatory solution $y(t)=(t-5)^{-t}$, which satisfies $\lim _{t \rightarrow \infty} y(t)=0$. 


\section{Forced oscillation}

THEOREM 3. Consider the functional differential equation (5), subject to the following hypotheses:

(H1) $p_{i}(t), r(t) \in C[[0, \infty), R],-p_{i}(t) \geqslant 0, i=1,2, \ldots, n$;

(H2) $g_{i}(t) \in C^{1}[[0, \infty), R], g_{i}(t)<t, \lim _{t \rightarrow \infty} g_{i}(t)=\infty, g_{i}^{\prime}(t) \geqslant 0, i=1,2, \ldots, n$;

(H3) $q(t)$ is continuous for any $t \neq 0$;

(H4) $f(y) \in C[R, R], y f(y)>0(y \neq 0), f(y)$ is nondecreasing and $f(x y)=f(x) f(y)$ for $x, y \in R$;

(H5) $\lim _{t \rightarrow \infty} \sup \sum_{i=1}^{n} \int_{G(t)}^{t}-p_{i}(s) f\left(\exp \left(\int_{c^{*}}^{g_{t}(s)} q(u) d u\right)\right) \exp \left(-\int_{c^{*}}^{s} q(u) d u\right) d s>M$,

where $G(t)=\max g_{i}(t), i=1,2, \ldots, n$, and $M$ is a positive constant;

(H6) there exists a function $Q(t) \in C^{1}[[0, \infty), R]$ such that

$$
Q^{\prime}(t)=r(t) \exp \left(-\int_{c^{*}}^{t} q(u) d u\right), \quad t \geqslant 0,
$$

and either

(I)

$$
\lim _{t \rightarrow \infty} Q(t)=0
$$

or

(II) there exist constants $q_{1}, q_{2}$ and sequences $\left\{t_{m}^{\prime}\right\},\left\{t_{m}^{\prime \prime}\right\}$ such that $\lim _{m \rightarrow \infty} t_{m}^{\prime}=\lim _{m \rightarrow \infty} t_{m}^{\prime \prime}=\infty$ and $Q\left(t_{m}^{\prime}\right)=q_{1}, Q\left(t_{m}^{\prime \prime}\right)=q_{2}, q_{1} \leqslant Q(t) \leqslant q_{2}$. $t \geqslant 0$.

Then, if (I) holds, every solution $y(t)$ of (5) is oscillatory or

$$
\lim _{t \rightarrow \infty} y(t) \exp \left(-\int_{c^{*}}^{t} q(s) d s\right)=0,
$$

while if (II) holds, every solution $y(t)$ of (5) is oscillatory or such that

$$
\lim _{t \rightarrow \infty}[z(t)-Q(t)]=-q_{1} \text { or }-q_{2} .
$$

Proof. Set $z(t)=y(t) \exp \left(-\int_{c^{*}}^{l} q(s) d s\right)$; then, by using the assumptions (H1), (H2), (H3) and (H4), (5) is obtained in the form

$$
z^{\prime}(t)+\sum_{i=1}^{n} l_{i}(t) f\left(z\left(g_{i}(t)\right)\right)=m(t),
$$

where

$$
l_{i}(t)=-p_{i}(t) f\left(\exp \left(\int_{c^{*}}^{g_{i}(t)} q(s) d s\right)\right) \exp \left(-\int_{c^{*}}^{t} q(s) d s\right)
$$


and

$$
m(t)=r(t) \exp \left(-\int_{c^{*}}^{t} q(s) d s\right) .
$$

We may suppose that $y(t)$ is a nonoscillatory solution of (5) and $y(t)$ is positive for sufficiently large $t$. In this case, $z(t)$ is also a nonoscillatory solution of (16) and $z(t)$ is positive for sufficiently large $t$. Put $Y(t)=z(t)-Q(t)$; then $Y(t)$ satisfies

$$
Y^{\prime}(t)+\sum_{i=1}^{n} l_{i}(t) f\left(Y\left(g_{i}(t)\right)+Q\left(g_{i}(t)\right)\right)=0 .
$$

From (17), because of (H1), (H2), (H4) and the fact that $z(t)=Y(t)+Q(t)>0$, we see $Y^{\prime}(t)<0$, so that we have $\lim _{i \rightarrow \infty} Y(t)=c$, where $c$ is a constant.

Suppose that the case (I) holds. If $c<0$, then we have the contradiction that $z(t)<0$ for sufficiently large $t$. If $c>0$, then we have

$$
z\left(g_{i}(t)\right)=Y\left(g_{i}(t)\right)+Q\left(g_{i}(t)\right) \geqslant c / 2, \quad i=1,2, \ldots, n,
$$

for sufficiently large $t$. From (17), we have

$$
Y^{\prime}(t)+\sum_{i=1}^{n} l_{i}(t) f(c / 2) \leqslant 0 .
$$

Integrating (18) from $G(t)$ to $t$, we have

$$
Y(t)-Y(G(t))+\left(\sum_{i=1}^{n} \int_{G(t)}^{t} l_{i}(s) d s\right) f(c / 2) \leqslant 0
$$

By taking the lim sup of (19), as $t \rightarrow \infty$, we get a contradiction to (H5). Hence, we conclude that $c=0$. From this, we see that

$$
\lim _{t \rightarrow \infty} z(t)=\lim _{t \rightarrow \infty} y(t) \exp \left(-\int_{c^{*}}^{t} q(s) d s\right)=0 .
$$

Suppose that the case (II) holds. Put $S(t)=Y(t)+q_{1}$. Then we have

$$
\lim _{t \rightarrow \infty} S(t)=\lim _{t \rightarrow \infty}\left(Y(t)+q_{1}\right)=c+q_{1} \equiv d \quad(-\infty<d<\infty) .
$$

If $d<0$ in (20), then we have $Y(t)+q_{1}<0$, for sufficiently large $t$, say $t \geqslant t_{1}$; this leads to a contradiction to the fact that

$$
Y\left(t_{s}^{\prime}\right)+q_{1}=Y\left(t_{s}^{\prime}\right)+Q\left(t_{s}^{\prime}\right)=z\left(t_{s}^{\prime}\right)>0, \text { for } t_{s}^{\prime}>t_{1} .
$$

If $d$ is positive, then we have

$$
z(t)=Y(t)+Q(t) \geqslant Y(t)+q_{1}=S(t)>d / 2
$$


for sufficiently large $t$, say $t \geqslant t_{2}$. By using (21) and (17), we get a contradiction to (H5). If $d=0$, then we have $\lim _{t \rightarrow \infty}[z(t)-Q(t)]=-q_{1}$.

Remark. Theorem 3 is connected to the results of Kusano and Onose (1974) and Tomaras's (1975b) Theorem 3.1.

\section{References}

Tosio Kato (1972), 'Asymptotic behaviour of solutions of the functional differential equation $y^{\prime}(x)=a y(\lambda x)+b y(x)$ ', Delay and functional differential equations and their applications, pp. 197-217 (Proc. Conf. Park City, Utah, 1972. Academic Press, New York, London, 1972).

Tosio Kato and J.B. McLeod (1971), 'The functional-differential equation $y^{\prime}(x)=a y(\lambda x)+b y(x)$ ', Bull. Amer. Math. Soc. 77, 891-937.

T. Kusano and H. Onose (1974), 'Oscillations of functional differential equations with retarded argument', J. Differential Equations, 15, 269-277.

G. Ladas, V. Lakshmikantham and J. S. Papadakis (1972), 'Oscillations of higher-order retarded differential equations generated by the retarded argument', Delay and functional differential equations and their applications pp. 219-231, (Proc. Conf. Park City, Utah, 1972. Academic Press, New York, London, 1972).

Alexander Tomaras (1975a), 'Oscillations of an equation relevant to an industrial problem', Bull. Austral. Math. Soc. 12, 425-431.

Alexander Tomaras (1975b), 'Oscillatory behaviour of an equation arising from an industrial problem', Bull. Austral. Math. Soc. 13, 255-260.

\section{Department of Mathematics}

Ibaraki University

Mito, 310

Japan 